\title{
Compact and Highly-Efficient Vertical Couplers for Active- Passive Monolithic Integration
}

\author{
M. Galarza, D. Van Thourhout and R. Baets \\ Department of Information Technology, University of Gent-IMEC, St.-Pietersnieuwstraat 41, B-9000 Ghent, Belgium \\ marko.galarza@intec.ugent.be \\ M. López-Amo \\ Departamento de Ingeniería Eléctrica y Electrónica, Universidad Pública de Navarra, Campus de Arrosadía, 31006 Iruña/Pamplona, Spain
}

\begin{abstract}
Ultra-compact low-loss vertical coupling between a $1.55 \mu \mathrm{m}$ InGaAsP bulk active waveguide and a passive waveguide based on bimodal interference is presented. Simulation results show polarization independence and good fabrication tolerances.

(2005 Optical Society of America

OCIS codes: (250.5300) Photonic integrated circuits; (130.1750) Components
\end{abstract}

\section{Introduction}

There is an increasing need for integrating multifunctional devices in a single chip in order to realize high performance components required in next generation optical communication systems. Planar monolithic integration is the way to integrate as much as possible the different active and passive functionalities of the opto-electronical component on a single substrate, resulting in small and compact units. It offers a significant reduction in production cost due to the batch processing of wafers.

Over the past years several active-passive integration techniques have been developed. Material regrowth [1], selective area growth [2] and quantum well intermixing [3] are complex and expensive technologies that result in high cost devices and/or low yields. Suematsu, et al. [4] proposed the twin-waveguide (TG) structure, where the active and passive functions are separated into two different vertically displaced waveguides, eliminating the need for material regrowth or any post-growth processing. Nevertheless, both waveguides have to be phase matched to transfer power by resonant coupling of the two supermodes, and a compromise in the device performance has to be made because the active and passive functionalities cannot be optimized separately.

In $[5,6]$ the authors propose the use of an asymmetric twin-waveguide (ATG) with tapered couplers. They utilize an active waveguide with a higher effective index than the passive waveguide, making them strongly asymmetric. Under this condition the even mode dominates in the active devices due to its larger gain and the odd mode is never excited. The asymmetric structure allows the independent optimization of the active devices. The power transfer to the passive waveguide is realized using a lateral tapering of the active waveguide. However, the authors in $[5,6]$ do not give a clear description of the power transfer process in the taper (adiabatic or modal interference), resulting in rather long designs $(\sim 150 \mu \mathrm{m})$ and sub-micron final taper tips $(\sim 0.5 \mu \mathrm{m})$, which are very difficult to achieve by standard lithography.

In this paper, we propose an approach for the monolithic integration of InGaAsP active and passive waveguides based on two physical phenomena: 1) power transfer from the fundamental mode to the first order mode of the ATG and 2) bimodal interference between both supermodes. This concept provides higher performance than the devices proposed so far, over a considerably shorter length and without the need of sub-micron lithography.

\section{Concept}

Fig. 1.a shows a simplified two dimensional representation of the adiabatic mode transformation produced in a tapered ATG (the lateral tapering of the real tapers is shown as a vertical tapering in the figure for clarity purposes). The mode generated in the upper active waveguide is in fact the even supermode $\psi_{e}$ of the complete structure formed by the active and the passive waveguides. In an adiabatic taper all the power remains in this mode while it is being transformed (see Fig. 1.a). The odd supermode $\psi_{o}$ supported by the structure is not excited in the adiabatic regime and when the tapered upper rib waveguide reaches cut-off due to the tapering, this mode $\psi_{o}$ is not a guided mode any more and becomes a radiation mode.

The mode transformation concept based on the bimodal interference between the two guided supermodes of the twin structure is schematically shown in Fig. 1.b. In region A all the power is carried by the even mode $\psi_{e}$. In region $\mathrm{B}$, an abrupt tapering of the waveguide provokes the transfer of part of the power from this fundamental even mode to the odd mode $\psi_{o}$ of the structure. In the straight section of region $\mathrm{C}$ the two modes propagate without loss and 


\section{IWG3}

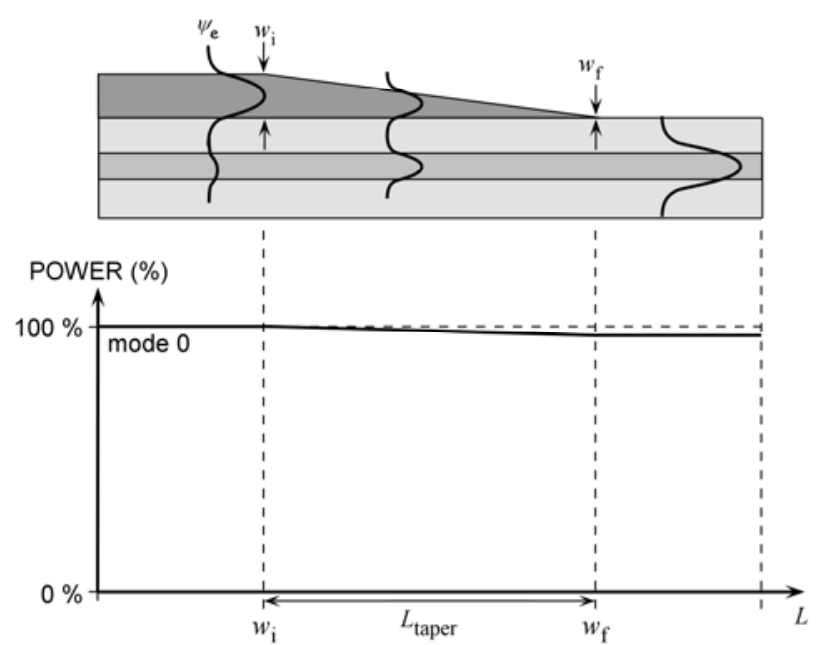

(a)

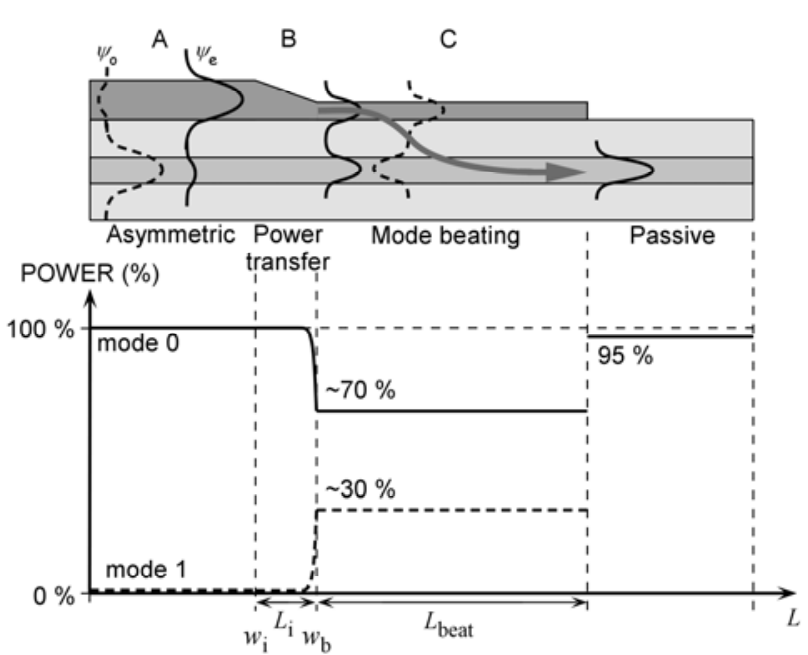

(b)

Fig. 1. (a) In the adiabatic mode transformation concept, the even supermode of the taper is smoothly transformed into the passive mode without loosing any power. (b) In the bimodal interference taper concept the fundamental supermode transfers power to the odd one and the interference between them couples the field in the passive waveguide.

interfere. At the beginning of region $C$ the field is concentrated in the upper active waveguide, but when the supermodes reach the coupling length (when their phase difference $\Delta \phi=\pi$ ), the power is concentrated in the lowerpassive waveguide and matches perfectly the mode of the individual passive waveguide.

The technique used to transfer power from the even eigenmode $\psi_{e}$ to the odd one $\psi_{o}$ can be understood by coupled mode theory. It is assumed that the transfer of power from the fundamental mode of a non-adiabatic taper will be predominantly to the higher order mode with a propagation constant closest to that of the fundamental mode [7]. Here this power-receiving mode is the odd supermode $\psi_{o}$. Therefore, by using a taper slope larger than the critical slope in region B we force the power transfer between the fundamental even mode $\psi_{e}$ and the odd mode $\psi_{o}$ [8]. Simulations show that this transfer can be performed without losing power to radiating modes.

\section{Design}

The ATG structure consists of a $200 \mathrm{~nm}$-thick and $2.5 \mu \mathrm{m}$-wide active bulk core of quaternary material for emission at $1.55 \mu \mathrm{m}(\mathrm{Q} 1.55)$ grown over a $500 \mathrm{~nm}$-thick interlayer of InP and a $375 \mathrm{~nm}$-thick passive core with an energy gap cutoff wavelength of $\lambda_{\mathrm{g}}=1.25 \mu \mathrm{m}$ (Q1.25). The width of the passive underlying waveguide is $3.4 \mu \mathrm{m}$ (see Fig. 2).

A commercial 3-D complex mode solver (Fimmwave) and a fully vectorial 3D propagation tool (Fimmprop) [9] were used to design the coupler. The initial width $w_{\mathrm{i}}$ is defined by the width of the active section, which is $2.5 \mu \mathrm{m}$. Therefore, the length $L_{\mathrm{i}}$ and the final width $w_{\mathrm{b}}$ of the sharp tapering, for which the top wave is synchronously coupled with the lower wavegude, have to be determined. At an active waveguide width $w_{\mathrm{b}}=1 \mu \mathrm{m}$, the effective indices of the two guides are closely
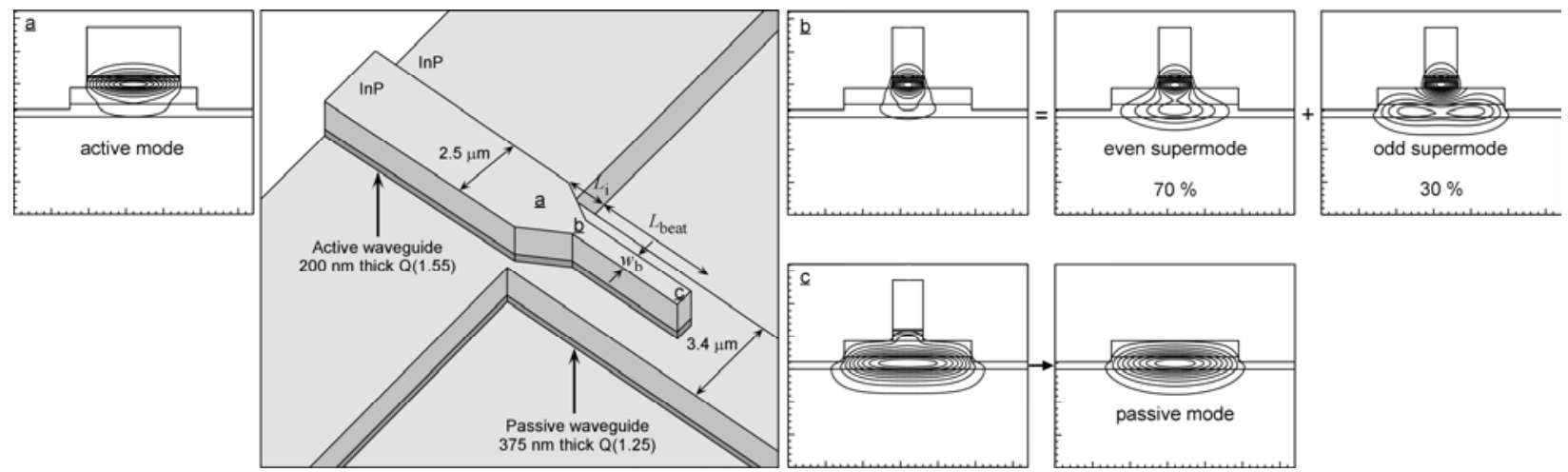

Fig. 2. Schematic diagram of the lateral tapering in a compact taper based on bimodal interference. Transversal field distributions for a propagation run at point $\underline{\mathrm{a}}, \underline{\mathrm{b}}, \underline{\mathrm{c}}$ are also shown. 


\section{IWG3}

matched, and the calculated beat length between the two supermodes is only $25 \mu \mathrm{m}$. No influence on the performance of the device is observed if $L_{\mathrm{i}}$ is maintained in the range $5-20 \mu \mathrm{m}$; therefore we choose a safe length $L_{\mathrm{i}}=15 \mu \mathrm{m}$, resulting in a total length for taper and couple section of $40 \mu \mathrm{m}$.

The structure in Fig. 2 is phase matched at the same width for both TE and TM polarizations, showing identical transformation efficiency. Therefore, the designed resonant coupler is polarization independent.

\section{Simulation results}

Fig. 2 shows the field distribution at three points in the taper during propagation. We observe how the field generated in the active waveguide keeps its shape in the upper waveguide at the end of the first section. This field distribution excites the two supermodes in the beating section in a rate of 70:30, coupling only $0.1 \mathrm{~dB}$ to radiation modes. As the two supermodes propagate in the straight section, they interfere and for the correct coupling length result in a field distribution that matches the passive mode, losing only $0.1 \mathrm{~dB}$ more.

Fig. 3 shows the efficiency of the proposed fully resonant coupler compared to the efficiency of an adiabatic design. We see that for the latter we need taper lengths longer than twice the length needed for the resonant coupler in order to reach comparable transformation losses $(<0.2 \mathrm{~dB})$.

We have performed a set of simulations to investigate the influence of changes in the width of the straight section $w_{\mathrm{b}}$. If the efficiency is taken as a figure of merit in this analysis we obtain a $1-\mathrm{dB}$ tolerance of $\Delta w= \pm 110$ $\mathrm{nm}$.

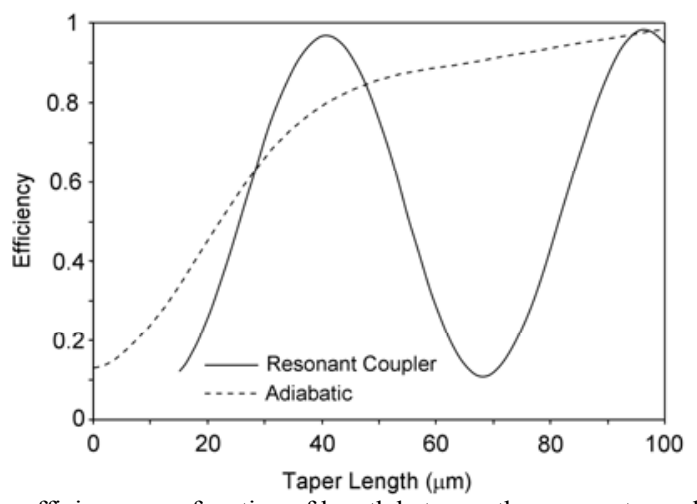

Fig. 3. Comparison of the taper efficiency as a function of length between the resonant coupling and adiabatic approaches.

\section{Conclusion}

In this work, we have presented highly efficient mode transformation in an ultra-compact device between an active waveguide and a passive waveguide using fully bimodal interference in an ultra-compact device. The method allows for separate optimization of the active and passive waveguide core. Modal transformation losses as low as $0.2 \mathrm{~dB}$ have been achieved in a polarization independent device using much shorter lengths than the adiabatic approach. The concept avoids submicron photolithographic features and shows acceptable fabrication tolerances.

\section{References}

[1] M.L. Masanovic, V. Lai, J.S. Barton, E.J. Skogen, L.A. Coldren and D.J. Blumenthal, "Monolithically integrated Mach-Zehnder interferometer wavelength converter and widely tunable laser in InP," IEEE Photon. Technol. Lett., 15, 1117-1119 (2003)

[2] T. Van Caenegem, D. Van Thourhout, M. Galarza, S. Verstuyft, I. Moerman, P. Van Daele, R. Baets, P. Demeester, C.G.P. Herben, X.J.M. Leijtens, M.K. Smit, "Monolithically integrated multi-wavelength laser by selective area growth with metal organic vapour phase epitaxy," Electron. Lett., 37, 296-298 (2001)

[3] J. Dubowski, Y. Feng, P. Poole, M. Buchanan, S. Poirier, J. Genest and V. Aimez, "Monolithic multiple wavelength ridge waveguide laser array fabricated by Nd:YAG laser induced quantum well intermixing," J. Vac. Sci. Technol. A, 20, 1426-1429 (2002)

[4] Y. Suematsu, M. Yamada and K. Hayashi, “Integrated twin-guide AlGaAs laser with multiheterostructure,” IEEE J. Quantum Electron., 11, (1975)

[5] S.S. Saini, F.G. Johnson, Z. Dilli, Y. Hu, R. Grover, D.R. Stone, P. Shen, J. Pamulapati, W. Zhou and M. Dagenais, “Compact low-loss vertical resonant mode coupling between two well-confined waveguides," Electron Lett., 35, 1195-1197 (1999)

[6] P.V. Studenkov, M.R. Gokhale and S.R. Forrest, "Efficient coupling in integrated twin-waveguide lasers using waveguide tapers," IEEE Photon. Tecnol. Lett., 9, 1096-1098 (1999)

[7] J.D. Love, W.M. Henry, W.J. Stewart, R.J. Black, S. Lacroix and F. Gonthier, "Tapered single-mode fibers and devices. Part 1: Adiabaticity criteria," IEE Proc.-J, 138, 343-354 (1991)

[8] M. Galarza, K. De Mesel, R. Baets, A. Martínez, C. Aramburu, M. López-Amo, "Compat spot-size converters with fiber-matched antiresonant reflecting optical waveguides," Appl. Opt., 42, 4841-4846 (2003)

[9] Fimmwave/Fimmprop, Photon Design; http://www.photond.com 